\title{
Image Retrieval using High Order Local Autocorrelation and MFCM
}

\author{
Meenakshi Srivastava ${ }^{1}$, Dr. S.K. Singh ${ }^{2}$ \\ Amity Institute of information Technology, Amity University, Lucknow, Uttar Pradesh, India ${ }^{1,2}$
}

\begin{abstract}
Time span has increased Dynamic Vast development of data like pictures, sound and video on web. Which has prompted established researchers for capacity and recovery of interactive media data all the more naturally? Since one picture can be translated from numerous points of view, catchphrase based seeking frameworks can recover the data to a restricted degree. This constraint has prompted the prerequisite for improvement of Content Based Image Retrieval Systems. Picture Retrieval frameworks which can perceive the articles like the human eyes have been generally prominent. To build a decent savvy vision framework, high measurement highlight vectors of pictures are required for example acknowledgment. In spite of the fact that high measurement include vectors gives mends facilitate with increment in number of pictures in database. In the present original copy creators have proposed a model which enough addresses these imperatives and gives a vigorous and quick recovery component of images.
\end{abstract}

Keywords: CBIR System, Image Retrieval, fuzzy C-mean Clustering (MFCM), High Order Local Autocorrelation.

\section{INTRODUCTION}

Boundless development of mixed media data on web has prompted mainstream researchers to create framework for capacity and recovery of interactive media data all the more naturally. The exploration in a decade ago demonstrates that recovery of mixed media data framework is obstructed by the "semantic hole", dwelling because of utilization of metadata/descriptors for clarifying the current substance in any interactive media question. Understanding of a picture should be possible through different routes; there is no institutionalized wording to speak to the substance of a picture through watchwords. Another method for speaking to any picture is by processing the interior components either via robotized strategy or by semi-mechanized procedure. In many fields of picture recovery robotized investigation ascertains a few insights which can further be utilized as a part of the request to figure the related substance highlights [1]. In spite of the fact that such calculations give required outcomes and a fulfilment level in many fields has been accomplished, yet a solitary system that fits best in a wide range of client's necessities is still to be created.

Hence, the entryways are as yet open to create new systems as per the necessities of image recovery applications [1][2]. The necessity of client for the most part relies on upon the unique situation and the application situation instead of the coordinating catchphrases as it were. Thus, it is especially required that the, mixed media processing systems understand, the content embedded in the multimedia data and recover the semantic structures as required by the end user. The conventional text based searching systems are able to facilitate retrieval in a limited sense. Due to this limitation, in past decades more attention has been directed towards techniques which can automatically recover the semantically meaningful structures from multimedia data like images. More emphasis has been put on retrieval algorithms which can recognize the objects like the human eyes [3] [4].

This development has motivated the researchers to design and develop the searching algorithms which can query and find the required information from these domain specific databases [6]. Various object recognition algorithms have been developed in recent years which follow the feature extraction and calculation of distance metrics in extracted feature to compute the similarity [5]. The high-dimension of feature vector is another factor which decreases the speed of image clustering and image retrieval [13][16]. Generally, the representation of feature vectors which leads to efficient and fast execution of algorithms are chosen against the more complex and computationally time-consuming approaches. Objective of present study is to develop an efficient system which will recognize the image as human eyes does [9]. In the present work, authors have focused on development of a new mechanisms which will aim at "querying" the databases of complex data sets by their content in terms of visual similarity, rather than by their textual annotations only. The prototype has been implemented as a "query-by example" system. The selected matching criteria are the visual features concerning the images themselves. Authors approach complements the traditional approach of querying the database by textual key words only.

The rest of the paper is organized as follows. In section II has discussed proposed model of object retrieval. In Section III Experimental details are presented. Result Analysis and Conclusion is done in section IV. 


\section{PROPOSED ALGORITHM}

One of the problems that is more frequent in the field of Multimedia is, answering the questions like "Which entities have the same structure as the given one?" This challenge still exists due to the reason of textual annotations/ metadata. The proposed algorithm takes into consideration the fact that our eyes can easily recognize two similar images by viewing them from various angels/ views. The proposed algorithm has been implemented and tested on CVonline datasets [17]. Our work focuses on retrieval of similar images by automatic feature extraction method. Authors have structured algorithm (Fig 1) into following five modules

(i) Query Interface Module

(ii) Geometrical Feature Extraction Module

(iii) Object Recognition Module

(iv) Image Retrieval and Visualization block.

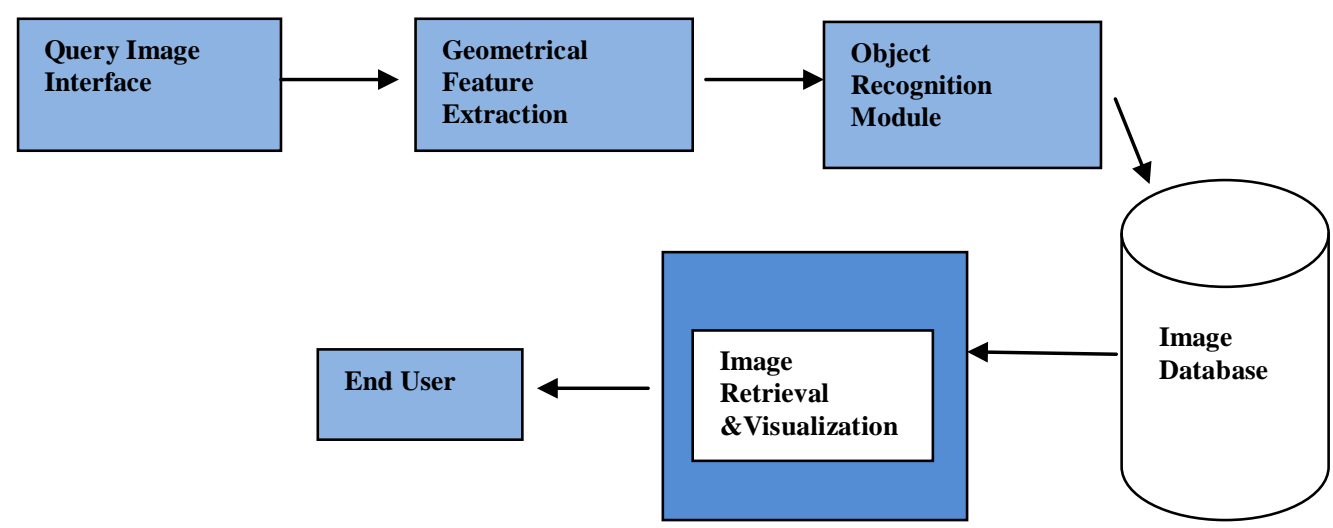

Fig 1. Modules of Algorithm: Query Image Interface Module, Geometrical Feature Extraction Module, Object Recognition Module, Image retrieval and visualization Module.

\subsection{Query Image Interface Module:}

Query Image interface has been implemented as Query by Example interface. The queried image is rotated on its axis and the High Order Autocorrelation Features are extracted from the image.

\subsection{Geometrical Feature Extraction Module:}

The size of the query image has been fixed to 256 × 256 pixels using MATLAB software.For geometrical feature extraction an intelligent vision algorithm proposed in [10][11]] has been deployed. Geometrical Feature Extraction concerns the extraction of features which are invariant under some transformation group acting on pattern [11]. The primitive features for an intelligent vision must be-

a. Shift Invariant - The empirical property of shift-invariance can be expressed using the following mathematical notation. Suppose a stimulus, pu and measure the system response, Ti. Now, shift the stimulus by an amount $\mathbf{j}$ and measure again. If the response is shifted by $\mathbf{j}$ as well, then the system may be shift-invariant. Try this experiment for many values of the shift parameter, $\mathbf{j}$. If the experiment succeeds for all shifts, then the system is shiftinvariant[7][8].

The autocorrelation function is shift Invariant, and its $\mathrm{N}$ order with $\mathrm{N}$ displacement $(\mathrm{a} 1, \mathrm{a} 2 \ldots ., \mathrm{aN})$ is defined as follows:

$$
\mathrm{x}(\mathrm{a} 1, \ldots, \mathrm{aN})=\sum \mathrm{I}(\mathrm{r}) \mathrm{I}(\mathrm{r}+\mathrm{a} 1) \ldots \mathrm{I}(\mathrm{r}+\mathrm{aN})
$$

Where $r$ is the image coordinate vector. The order $N$ is limited to the second order $(N \in\{0,1,2\})$.

b. $\quad$ Additive - $\mathrm{z}[\mathrm{f}]$ is additive, is represented by

$$
\mathrm{X}[\mathrm{f} 1=\mathrm{f} 2]=\mathrm{x}[\mathrm{f} 1]=\mathrm{x}[\mathrm{f} 2] \text { for Supp (f1) } \cap \operatorname{Supp}(\mathrm{f} 2)=\varnothing .
$$

Each supplied query image is rotated randomly around its three principal viewing axes and multiple-views of 2D images are stored. 2D HLAC (High Order Local Autocorrelation) features [10] are extracted from the query images. Duplicate configurations of $\mathrm{r}, \mathrm{r}+\mathrm{a} 1, \ldots, \mathrm{r}+\mathrm{a} \mathrm{N}$ are removed and local mask patterns are reduced to 35 .

\subsection{Object Recognition Module:}

80 images were randomly collected from the CVOnline dataset[17]. Combining the extracted primitive features were the next step for pattern recognition, adaptability and trainability. This leads to use of multivariate data analysis methods. In multivariate data analysis methods, new features are given by linear combinations of the primitive features. 
The high-dimension feature vector leads to decrease the speed of image clustering and image retrieval. To reduce the dimension of feature vector Principal Component Analysis is performed on the HLAC feature vector. PCA performs a linear mapping of the data to a lower-dimensional space in such a way that the variance of the data in the lowdimensional representation is maximized. While computing the PCA first the covariance/ correlation matrix of the feature vector is constructed and then Eigen vectors on covariance matrix are computed. The principal components are those Eigen vectors that correspond to the largest Eigen values. Practically it has been seen that the first few Eigen vectors can be used to represent the system. The clustering is performed through modified fuzzy C-means (MFCM) clustering index algorithm [13]. MFCM [13] algorithm has been used to cluster the high dimensional data in the database. By applying MFCM database can be classified into K clusters and each cluster will be represented by a clustering center. Each image will be assigned to a cluster where the distance between image and clustering center is the closest of all [16]. After classification, the similar images will be assigned to the same cluster. The most important step during the whole progress of MFCM algorithm is selection of reasonable initial center. The number of initial centers is selected as the formula defined in [14][15].

\subsection{Image Retrieval\& Visualization Module:}

In order, searching of image database always results in enormous computations which are very time consuming. By classifying all images in database according this indexing structure, image searching scope has been reduced greatly which has fastened the retrieval rate. Retrieval speed is an important issue in image retrieval system, while image information is abundantly, and the data of image database is large [13][16].One more advantage of clustering index scheme is that the increasing number of feature dimensions and the size of image database time of image retrieval won't increase in linear proportion. The retrieved image set has been visualized and shown to the end user in decreasing order of similarity index.

\section{EXPERIMENTAL RESULTS}

The effectiveness of the proposed method has been evaluated on images collected randomly from the CVOnline dataset[17]. 80 images were collected, Matlab [15] was used to resize the image has been rotated on its prime axis and multiple views of images have been stored. 2000 images for each image have been synthesized. The color scheme has been set to gray-scaled.

HLAC features have been extracted from the multi view images. In the Nth order displacement the value of $\mathrm{N}$ has been fixed to 2. Combined High Order Local Autocorrelation feature vector of 35 dimensions has been generated. On image feature matrix $\times[\mathrm{M} \mathrm{x} \mathrm{N}]$, eigenvector corresponding to the absolute maximal Eigen value of the covariance of matrix $\mathrm{x}[\mathrm{M} \square \square \mathrm{N}]$ has been calculated. The dimensions of the reference Subspace (DataBase) and the Input subspace (Query Image) were varied .MFCM clustering index has been applied to the high dimensional feature vector. Prototype of the model is implemented in MATLAB [15].

\section{RESULT ANALYSIS CONCLUSION AND FUTURE WORK}

In order to test the validity and efficiency of the proposed algorithm MFCM method is compared with MSM based clustering.

Table 1. Comparison of Precision and Retrieval time

\begin{tabular}{|c|c|c|}
\hline & MSM based k-means clustering & MFCM Clustering \\
\hline Precision & 98.78 & 96.19 \\
\hline Recall & 97.23 & 95.3 \\
\hline Retrieval time (ms) & 106.32 & 94.73 \\
\hline
\end{tabular}

Table1 shows that compared with in order retrieval, MFCM has the fast retrieval speed than Mutual Sub Space based kmeans clustering. $95 \%$ recall rate and $96 \%$ precision rate at a very fast speed was achieved.

As discussed earlier, one of the main problems associated with retrieval of images is the regular growth of new images in database and slow retrieval due to online similarity comparison. Our results show that if the number of images is increased in the database then also the time of retrieval doesn't increase linearly with them

Model proposed can efficiently retrieve images in faster manner. Prototype model has been implemented in MATLAB. Approach is based on retrieving the images which are visually similar. This problem has been overcome by applying MFCM clustering index algorithm. Experimental results show that increase in number of images in data base does not linearly effect the retrieval time. Presently performed tests are on very small data set, in the future work more data will be collected and clustering on them will be performed. 


\section{REFERENCES}

[1] Mussarat Yasmin, Sajjad Mohsin, Muhammad Sharif, Intelligent Image Retrieval Techniques: A Survey, Journal of Applied research and technology, Vol 14 December 16.

[2] Meenakshi Srivastava, Dr. S.K. Singh, Dr. S.Q. Abbas, "Web Archiving: Past Present and Future of Evolving Multimedia Legacy", International Advanced Research Journal in Science, Engineering and Technology Vol. 3, Issue 3.

[3] Meenakshi Srivastava, “A Query by Example Approach for Retrieving 3D Images Based on Their Visual Similarity”, International Journal of Advanced Research in Computer and Communication Engineering Vol. 5, Issue 5, May 2016

[4] Shape modeling international 2004, pp 157-166P. Kumswat, Ki. Attakitmongcol and A. Striaew, "A New Approach for Shen Y-T, Chen D-Y, Tian X-P, Ouhyoung M (2003) 3D model search engine based on

[5] Shen Y-T, Chen D-Y, Tian X-P, Ouhyoung M (2003) 3D model search engine based on light field descriptors.In: Proc. eurographics 2003.

[6] Kazhdan M, Funkhouser T, Rusinkiewicz S (2004) Shape matching and anisotropy. In: Proc. SIGGRAPH 2004

[7] Körtgen M, Park G-J, Novotni M, Klein R (2003) 3D shape matching with 3D shape contexts. In: Proc. $7^{\text {th }}$ central European seminar on computer graphics, April.

[8] Mamic G, Bennamoun M (2002) Representation and recognition of 3D free-form objects. Digit Signal Process 12:47-67

[9] Min P (2004) A 3D model search engine. PhD thesis, Princeton University.

[10] Motofumi T. Suzuki, Texture Image Classification using Extended 2D HLAC Features,KEER2014, LINKÖPING | JUNE 11-132014 International Conference On Kansai Engineering And Emotion ResearchTexture

[11] Nobuyuki OTSU and Takio Kurita, A New Scheme for Practical Flexible And Intelligent Vision Systems, 1APR Workshop on CV -Special Hardware and Industrial Applications OCT.12-14, 1988, Tokyo.

[12] A. Herr'aez. Biomolecules in the computer: Jmol to the rescue. Biochemistry and Molecular Biology Education,

[13] Liu Pengyu, Jia Kebin, Lv Zhuoyi, An Effective and Fast Retrieval Algorithm for Content-based Image Retrieval, 2008 Congress on Image and Signal Processing

[14] Kouassi R., Gouton P., Paindavoine M.: “Approximation of the Karhunen- Loeve transformation and its application to color images”, Signal Processing: Image Communication, vol. 16, 2001, pp. 541-551.

[15] MATLAB and Statistics Toolbox Release 2014b, The MathWorks, Inc., Natick, Massachusetts, United States.

[16] Zhuoyi Lv. "An Effective and Fast Retrieval Algorithm for Content-Based Image Retrieval", 2008 Congress on Image and Signal Processing, $05 / 2008$

[17] http://homepages.inf.ed.ac.uk/rbf/CVonline/Imagedbase.htm. 